\title{
24
}

\section{Machining feature generation based on a facility model and manufacturing knowledge}

\author{
M. Matsuda \\ Department of Information and Computer Sciences \\ Faculty of Engineering, Kanagawa Institute of Technology \\ 1030 Shimo-ogino, Atsugi-shi, Kanagawa 243-02, Japan \\ Telephone:81-462-41-1211, Fax:81-462-42-8490 \\ E-mail:matsuda@ic.kanagawa-it.ac.jp \\ F. Kimura \\ Department of Precision Machinery Engineering \\ Graduate School of Engineering, The University of Tokyo \\ 7-3-1 Hongo, Bunkyo-ku,Tokyo 113, Japan \\ Telephone:81-3-3812-2111, Fax:81-3-3812-8849 \\ E-mail:kimura@cim.pe.u-tokyo.ac.jp
}

\begin{abstract}
The derivation process of a product model for machining through the generation of machining features is discussed in this paper. Descriptions of the machining features are derived by applying machining constraints to the product model for design. Machining constraints consist of machine specifications, machine functions, and machining knowledge. Here the machine specification and functions are represented as a machine model, which is a type of facility model. The machine model is composed of a specification model and a process model. The specification model is the description of the static elements of the machine. The process model is the description of the actual operation by means of simulation. Manufacturing knowledge is used for listing candidates of machining methods, using tools, operation order, and machining conditions. A prototype system for milling feature generation using the milling machine model and machining knowledge is provided.
\end{abstract}




\section{Keywords}

Product modeling, machining feature description, machine model, virtual machining, machining preparation

\section{INTRODUCTION}

In a concurrent manufacturing system, a product model is used at various stages, such as product design and process/operation planning. However, the required product model differs depending on the stages. For example, a product model with machining feature description is required for the machining preparation or checking the process for machinability. This means that there is a product model for design, a product model for machining, a product model for assembly, and so on. In some cases, a product model for design is a product model with design feature descriptions, and a product model for machining is a product model with machining feature descriptions. In a concurrent manufacturing framework, all product models for XXX should be transformable between themselves, since all product models for XXX should be commonly usable as one product model at every manufacturing stage. Therefore, a framework for handling the product model is required, as pointed out in the literature. (Kimura, 1993) (Krause, 1993). This framework will provide flexible use and operation for product data, and as such, will provide one of the basic techniques for achieving concurrent manufacturing.

Usually, a product model is created at the design stage. At this stage, the product model is described using design features. When this product model with design features was automatically transformed to the product model with machining features, a total automatic machining system from product design to actual machining was realized (Matsuda, 1991). This transformation mechanism showed that descriptions of machining features were derived by applying machining constraints, such as usable machines and tools, to descriptions of design features (Matsuda, 1995) (Matsuda, 1996). A general methodology for handling the product model will be provided by expansion of this mechanism. This paper tries to provide the methodology which derives the product model for machining from the product model for design. Finally, this paper offers a proposal for the basic framework for handling the product model using manufacturing environment models. 


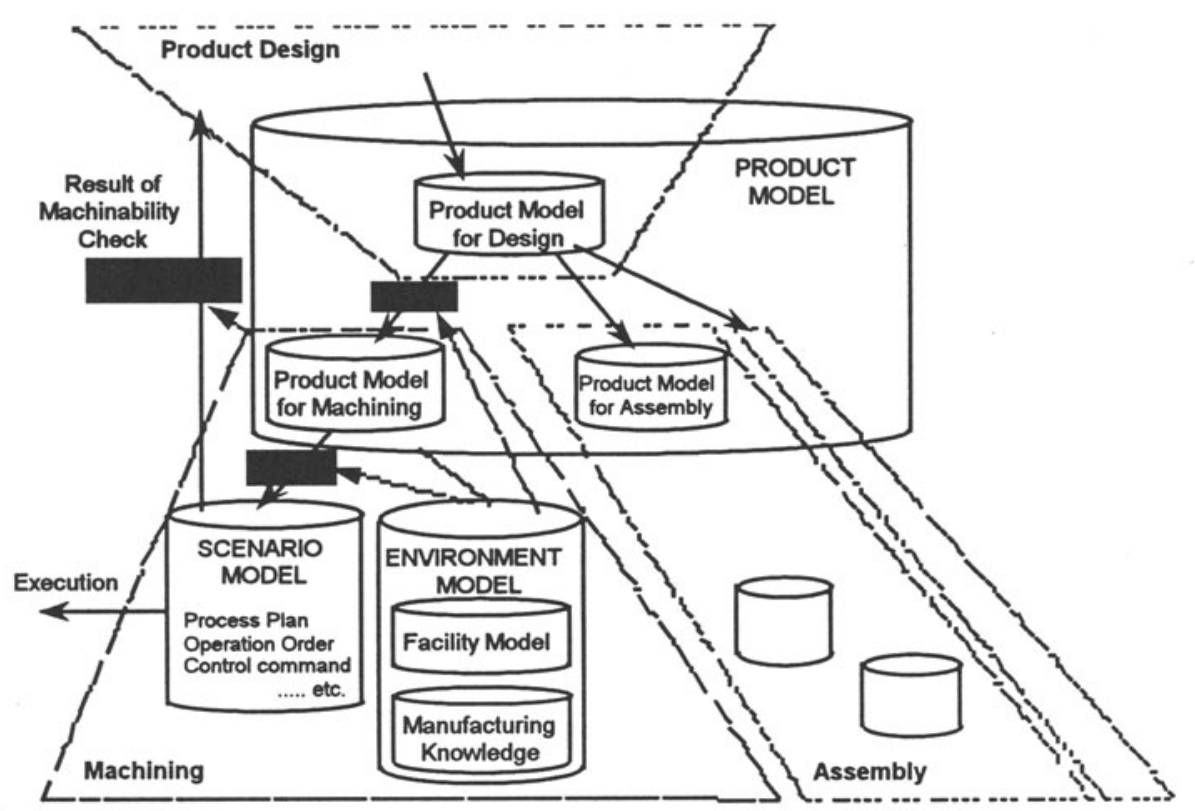

Figure 1 Product model, scenario model and environment model.

\section{INTEGRATION FRAMEWORK FOR PRODUCT, SCENARIO AND ENVIRONMENT MODELS}

At the machining or assembly process/operation planning stage, it is required to focus on features which relate to operation units, such as a volume machined by a single tool or attaching faces at the assembly stage. The product model for machining or the product model for assembly is a product model which has explicit descriptions of adequate features. Similarly, there are product models for measuring, product models for quality control, and so on.

Usually, a computer system generates a machining or assembly process plan, the order of operation, operation condition and control commands are determined by using the above mentioned feature descriptions. These generated plans, operation sequences, conditions and commands are represented as scenario models. The scenario model is used not only for actual production but also for simulation or evaluation. In order to realize a concurrent manufacturing system, the first step is the generation of a product model with adequate feature descriptions based on the product model used at the product design stage, and then generate a scenario model based on a suitable product model.

Descriptions of adequate features, such as machining features or assembly features, are derived by consideration of the target facility, such as machines, tools, or robots used. Manufacturing knowledge is used when making decisions on selecting a target facility. In other words, the facility information and knowledge are used as manufacturing constraints for the derivation of feature descriptions. 
Here, these manufacturing constraints are represented as an environment model. The environment model consists of facility models and manufacturing knowledge.

Figure 1 shows the relationship between the product model, the scenario model, and the environment model. If the designer wants to check for machinability or assemblability of the product, the following steps have to be executed. First, the system has to generate a product model with machining or assembly feature descriptions by using the environment model. Second, the system will have to generate a scenario model based on the product model while utilizing the environment model. Third, the system will have to simulate the scenario model and evaluate the result by again using the environment model. In order to achieve the actual production, the process which generates the scenario model via the product model including adequate feature descriptions will have to be executed. The environment model is required at every process stage.

\section{ENVIRONMENT MODEL}

\subsection{Facility model}

The facility model models the static and dynamic properties of equipment. As shown in Figure 2, a facility model has a hierarchical structure. At the highest level is the equipment model such as a machine model or a robot model. At the middle level are models of units, which are components of the equipment. A unit is one functional block such as a controller and mechanism in the machine, or an arm and hand in the robot. At the lowest level are the models of parts which compose a unit.

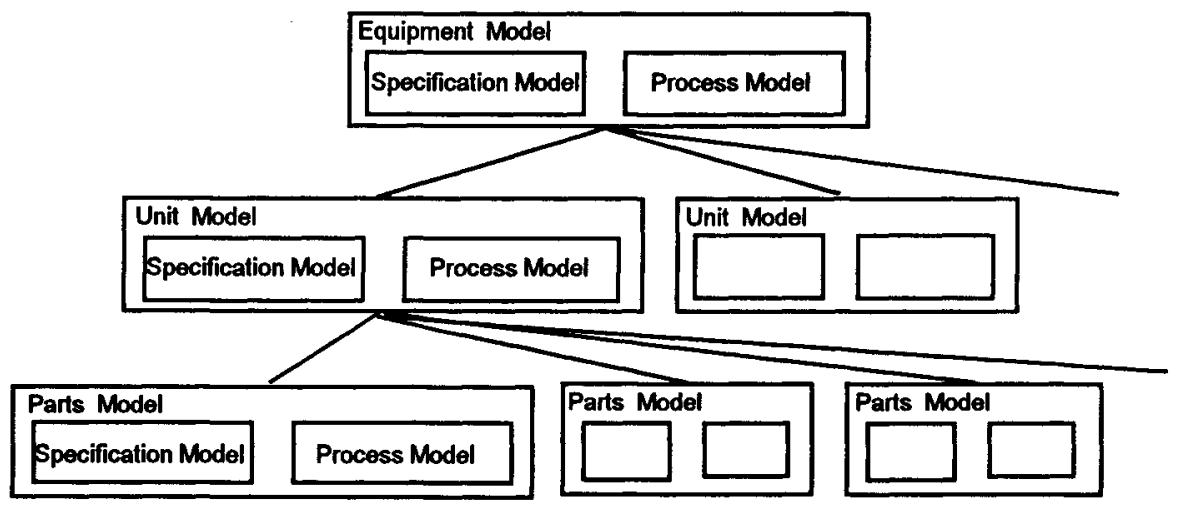

Figure 2 Structure of facility model. 


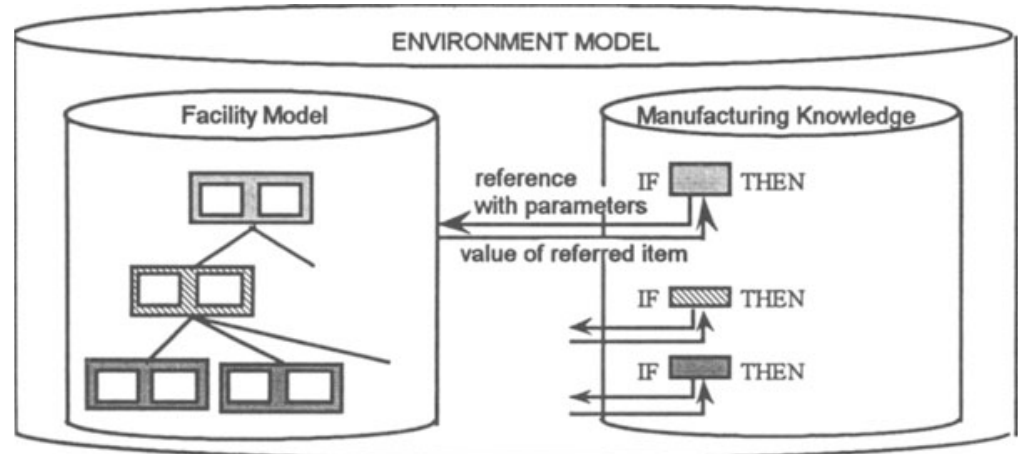

Figure 3 Structure of environment model.

A part is one element of a unit such as a tool or a robot finger. In some cases, a facility model may have more or fewer levels in the hierarchy.

Each of the equipment, unit and part models are composed of a specification model and a process model. The specification model consists of a description of static data, such as name, type, size, speed, and weight. The process model consists of a description of dynamic data, such as the results of some operation or condition during an operation. The process model at the equipment level and unit level has itself a hierarchical structure, since the total behavior of the equipment is based on the combined behavior of the motion of its units, and similarly, the behavior of a unit is totally the result of the motion of its parts. In other words, the process model represents the function of the equipment, units and parts. The implementation of the process model includes a simulation process.

\subsection{Manufacturing knowledge}

Manufacturing knowledge is used for listing candidates for a manufacturing method using a machine and process/operation plan. Also manufacturing knowledge is used for decision making when selecting from the candidates and generating the actual operation order and control commands. Manufacturing knowledge consists of manufacturing know-how, decision rules, the basis for evaluation and so on. As shown in Figure 3, the manufacturing knowledge description has a hierarchical structure corresponding to the hierarchy of the facility model. Most manufacturing knowledge is described by IF-THEN rules. When applying a rule, the actual value for the condition of a part is provided by reference to the facility model at the corresponding level. For example, when selecting a device to be used, the equipment model is referred to, with parameters such as device identifier, product description, and reference item. The reference item indicates what kind of value is desired in this reference. 


\section{DERIVATION OF PRODUCT MODEL FOR MACHINING}

In this paper, a product model for machining means a product model with machining feature descriptions. Similarly a machining feature means, for example, the volume which is machined by a single tool. Figure 4 shows the basic derivation process for the product model with machining features by using the machining environment model. All machines and tools in the factory are modeled as facility models in the machining environment model. Also, the machining knowledge is described in the machining environment model. The product description already exists as a product model for design. The derivation proceeds as follows:

1) Candidates of applicable machines are generated and listed. Under considerations of product shape, size, material, required finished accuracy, and so on, the system selects usable machines by referring to the machining environment model.

2) The machine for the actual production is selected, taking account of machining cost, schedule, and so on. While the decision process is taking place, the environment model, especially the process model and knowledge, are referenced.

3) Candidates of applicable tools are generated and listed, taking into consideration the product shape, product size, finished accuracy, cutting stage and so on. The system makes reference to tool models and related knowledge in the environment model.

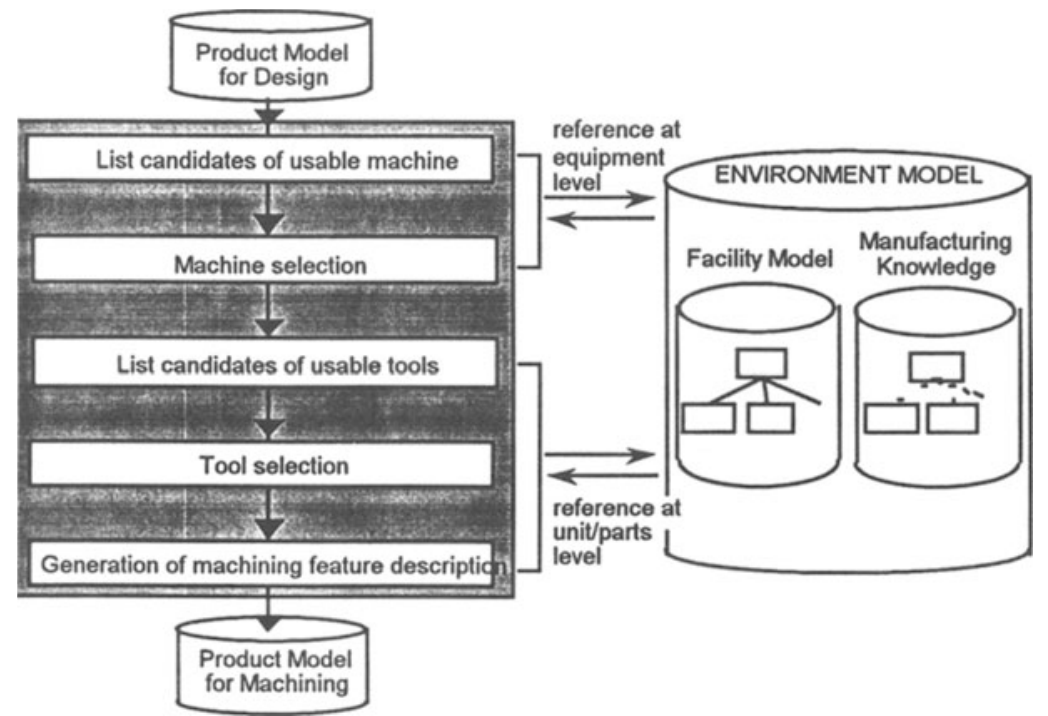

Figure 4 Derivation process of machining feature.

4) The tools to be used are selected through rejection of candidate tools which have low cutting efficiency, such as a small machining area or long cutting time. During 
the rejection process, process models for each tool model and knowledge are referenced.

5) Machining features are generated by means of calculations of machined volumes

corresponding to each of the selected tools.

\section{MILLING DATA GENERATION SYSTEM}

\subsection{Structure of the system}

The milling data generation system described is a prototype system under development by the authors. This system produces a process plan for cutting as well as NC data based on the derived milling features. A milling feature is one type of machining feature. This system considers milling of a cavity for a die or mold. A cavity is one kind of a pocket. This system treats only a pocket with a complicated 2 1/2 dimensional shape. A $21 / 2$ dimensional shape means the combination of several volumes which are derived by sweeping a 2 dimensional figure. Usually, straight endmills are used for $21 / 2$ dimensional shape cutting.

The structure of this system is shown in Figure 5. This system consists of two parts. One is the milling feature generator, and the other is the milling data generator. The product description which is obtained at the product design stage, provides inputs to the milling feature generator. In the milling feature generator, the milled cross section as milling area is determined based on the product shape. This is followed by the milling feature generation and tool selection, based on the milled cross section and making use of the milling environment model. Finally,

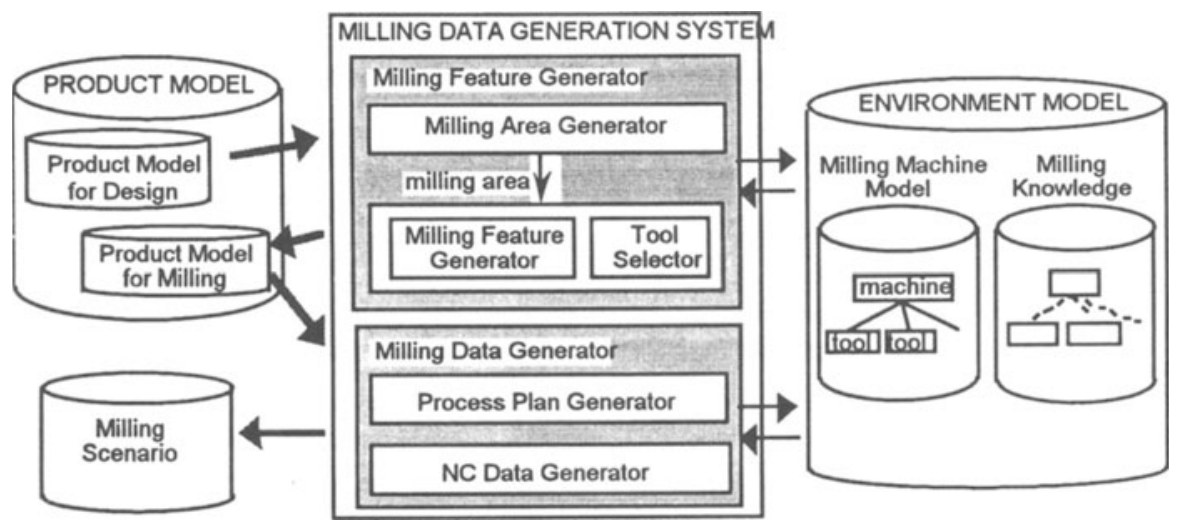

Figure 5 Milling feature generation system. 


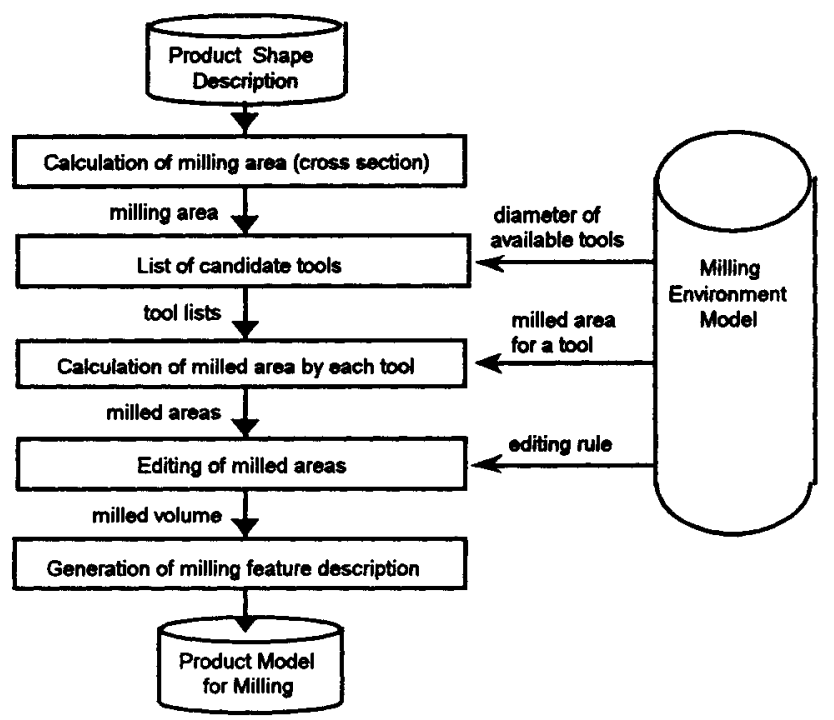

Figure 6 Process of milling feature generation.

the milling feature description is provided. The milling environment model consists of the milling machine model and milling knowledge. The milling machine model has a two level hierarchy. The higher level is at the milling machine level, while the lower level is at the tool level which is a description of available tools. The milling data generator produces a cutting process plan and NC data as a milling scenario based on generated milling features by using the milling environment model.

\subsection{Milling feature generation}

The process flow for milling feature generation is shown in Figure 6. The generation process proceeds as follows:

1) The milled cross section at each bottom face depth is computed. Each milled cross section is considered independently as a milling area.

2) For one milling area, the candidate list of possible tools are selected in the form of tool diameters of a straight endmill. This selection process is based on the corner size, distance between island areas, and the diameter of the biggest available tool. List of available tools and selection rules are provided by the milling environment model. The final candidate list of applied tool diameters consists in an ordering of tools according to their diameters from largest to smallest, since the largest possible tool would provide optimum milling efficiency.

3) For each milling area, the list of milled areas are calculated by applying each tool on the corresponding candidate list. The milled area is the machinable 2 
dimensional figure cut by the diameter of the candidate tool. The milled area is provided from the process model of the milling machine model with reference to the corresponding milling area and applied tool diameter. The result of this stage is a list of resulting milled areas, and a list of applied tools, ordered according to their diameters.

4) The milled areas are edited according to editing rules in order to obtain milled volumes. In each list of milled areas, the milled area for the bigger tool is subtracted from the milled area for the smaller tool. The result of the subtraction provides the actual milled area for the smaller tool, because actual cutting starts with the bigger tool. The result is registered as the correct milled area for the smaller tool on the list of milled areas. However, if the result of the subtraction is smaller than the threshold area, the milled area for smaller tool is eliminated from the list. The threshold value is decided based on the milling knowledge. Milled areas on all lists are divided into groups according to tool diameter. These groups of tools are ordered by size, largest to smallest. In one group, milled areas are ordered by depth, shallow to deep, and duplicated milled areas at each depth are eliminated. The volume cut by a single tool is obtained by sweeping the milled areas for one tool.

5) One milled volume corresponds to one milling feature. Milling feature descriptions are derived from the milled volumes.

\section{CONCLUSIONS}

A mechanism for deriving machining feature descriptions using a milling environment model was proposed, based on the concept that machining features are obtained by applying machining constraints to the product model for design, and that machining constraints are represented as an environment model. A prototype system developed showed the basic propriety and utility of the proposal.

Through using an environment model which is composed of facility models and manufacturing knowledge, the flexibility and expandability of the proposed system are increased. For example, when a new machine is added in a factory, only the addition of a new machine model and its related knowledge is required. Furthermore, it is possible to compare machinability by generating and simulating machining data for corresponding available machines, since the system has several facility models available at the same time. This means that the proposed system is one kind of a virtual manufacturing system.

\section{REFERENCES}

Kimura, F. (1993) Product and Process Modeling as a Kernel for Virtual Manufacturing Environment: Annals of CIRP, Vol.42, No.1, 147-150.

Krause, F.-L., Kimura, F., Kjellberg, T. and Lu, S.C.-Y. (1993) Product Modeling: Annals of CIRP, Vol.42, No.2, 695-706. 
Matsuda, M. and Kimura, F. (1991) Extraction of machining feature for milling data generation: Proceeding of CAPE'91. IFIP TC5, North-Holland, 353-360.

Matsuda, M. and Kimura, F. (1995) Modeling of virtual manufacturing devices for machining data generation: Proceeding of CAPE'95.IFIP TC5, Chapman \& Hall, 407-414.

Matsuda, M. and Kimura, F. (1997) Generation of milling data in a virtual manufacturing framework: The Design of Information Infrastructure Systems for Manufacturing, IFIP TC5, Chapman \& Hall, in print.

\section{BIOGRAPHY}

M.Matsuda is an associate professor in the Department of Information and Computer Sciences of Kanagawa Institute of Technology. She received a Dr.Eng. degree in precision machinery engineering from the University of Tokyo in 1989. She was an associate professor in the Department of Computer Science of Sanno Institute of Management from 1991 to March 1997. She has been active in the field of $\mathrm{CAD} / \mathrm{CAM}$, process/operation planning, NC programming and numerical control. Her recent research interests include product modeling for manufacturing and virtual manufacturing. She is a member of Japan Society of Precision Engineering and Information Processing Society of Japan.

F.Kimura is a professor in the Department of Precision Machinery Engineering of the University of Tokyo. He received a Dr. Eng. Sci. degree in aeronautics from the University of Tokyo in 1974. He was a research associate at the Electroacoustics Laboratory of the Ministry of International Trade and Industry from 1974 to 1979 . He then moved to the University of Tokyo, and was an associate professor from 1979 to 1987 . He has been active in the field of solid modeling, free-form surface modeling and product modeling. His research interests now include the basic theory of $\mathrm{CAD} / \mathrm{CAM}$ and $\mathrm{CIM}$, concurrent engineering, engineering simulation, virtual manufacturing and life-cycle engineering. He is a member of IFIP WG5.2 and WG5.3, and a member of CIRP. 